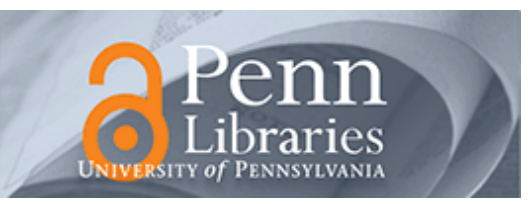

University of Pennsylvania

ScholarlyCommons

July 2001

\title{
Do What I Mean: Online Shopping with a Natural Language Search Agent
}

\author{
Barry G. Silverman \\ University of Pennsylvania, basil@seas.upenn.edu \\ Mintu Bachann \\ Equidity \\ Khaled Al-Akharas \\ Equidity
}

Follow this and additional works at: https://repository.upenn.edu/ese_papers

\section{Recommended Citation}

Barry G. Silverman, Mintu Bachann, and Khaled Al-Akharas, "Do What I Mean: Online Shopping with a Natural Language Search Agent", . July 2001.

Copyright 2001 IEEE. Reprinted from IEEE Intelligent Systems, Volume 16, Issue 4, July/August 2001, pages $48-53$.

Publisher URL: http://ieeexplore.ieee.org/xpl/tocresult.jsp?isNumber=20376\&puNumber=5254

This material is posted here with permission of the IEEE. Such permission of the IEEE does not in any way imply IEEE endorsement of any of the University of Pennsylvania's products or services. Internal or personal use of this material is permitted. However, permission to reprint/republish this material for advertising or promotional purposes or for creating new collective works for resale or redistribution must be obtained from the IEEE by writing to pubs-permissions@ieee.org. By choosing to view this document, you agree to all provisions of the copyright laws protecting it.

This paper is posted at ScholarlyCommons. https://repository.upenn.edu/ese_papers/42

For more information, please contact repository@pobox.upenn.edu. 


\title{
Do What I Mean: Online Shopping with a Natural Language Search Agent
}

\author{
Abstract \\ Ineffective search engines on e-catalog sites are driving away potential customers. Natural-language \\ querying improves precision and parsing capability, and with advances in the technology, it can also meet \\ these shopping sites' performance demands.

\section{Comments} \\ Copyright 2001 IEEE. Reprinted from IEEE Intelligent Systems, Volume 16, Issue 4, July/August 2001, \\ pages 48-53. \\ Publisher URL: http://ieeexplore.ieee.org/xpl/tocresult.jsp?isNumber=20376\&puNumber=5254 \\ This material is posted here with permission of the IEEE. Such permission of the IEEE does not in any way \\ imply IEEE endorsement of any of the University of Pennsylvania's products or services. Internal or \\ personal use of this material is permitted. However, permission to reprint/republish this material for \\ advertising or promotional purposes or for creating new collective works for resale or redistribution must \\ be obtained from the IEEE by writing to pubs-permissions@ieee.org. By choosing to view this document, \\ you agree to all provisions of the copyright laws protecting it.
}


Ineffective search

engines on e-catalog

sites are driving away

potential customers.

Natural language

query improves

precision and parsing

capability, and with

advances in the

technology, it can also

meet these shopping

sites'performance

demands.

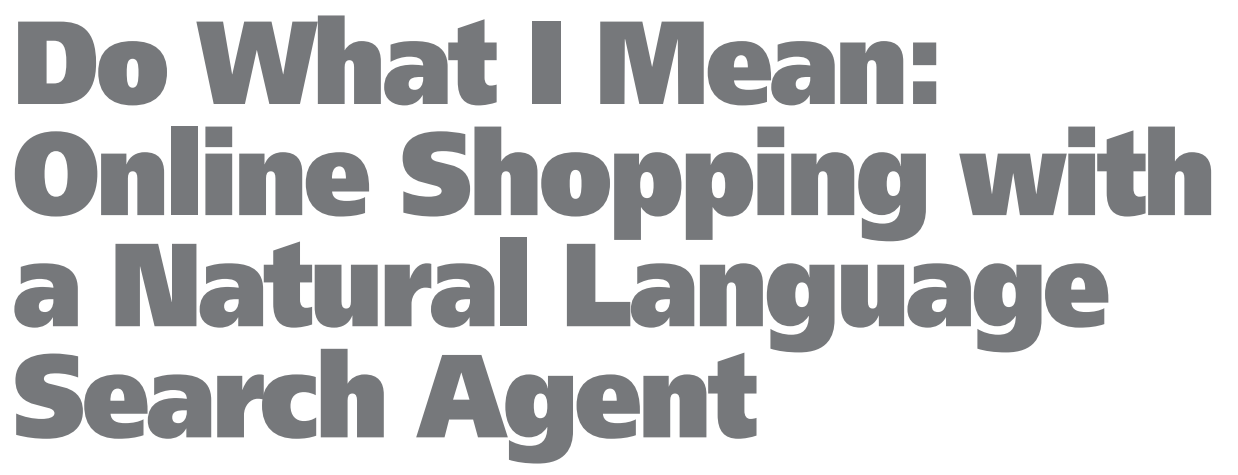

Barry G. Silverman, University of Pennsylvania

Mintu Bachann and Khaled Al-Akharas, Equidity

$\mathbf{M}$ arket exchanges are Internet sites that virtually integrate multiple vendors' catalogs. They let users browse, search for, bid on, finance, buy, or ship products. Such sites-Amazon.com and Ariba.com are two examples among manyconstitute a major form of e-commerce in both the business-to-consumer and business-

to-business segments. Over half of today's 80 million Web users shop for or buy products online, and analysts expect B2B purchasing to eclipse that level rapidly. ${ }^{1}$

However, in the rush to establish an online presence, many enterprises have built their e-market sites with little infrastructure and few of the capabilities necessary for such an e-business. For example, in March 2000, the Boston Consulting Group reported that more than 80 percent of Web shoppers have at some point left e-markets without finding what they wanted and that 23 percent of all attempted e-shopping transactions end in failure. ${ }^{1}$ Four of the top five failure modes are search-related-long page-loading times, failures to find the product, system crashes, and calls to customer service.

Research also shows that about 15 percent of online search failures stem from spelling errors and another 40 percent result from customers using different terms from those in the Web site ${ }^{1,2}$-for example, using "patching" when the Web site uses "concrete." Because keyword-based search engines can't interpret the meaning of users' queries, they typically bring back innumerable hits of everything even remotely relevant, often burying the best choice deep within the list or omitting it altogether. On top of that, such search engines seldom help the shopper narrow down the returned set of items.
Two approaches exist for improving keywordbased search: conceptual query (CQ) and natural language query (for a comparison, see the sidebar). This article describes an agent that uses restricted natural language to help users search product catalogs in large-scale market exchanges.

\section{The challenges}

Before we turn to our NLQ search, let's examine the challenges that catalogs pose for any search method. Specifically, in shopping site searches, one challenge is handling incomplete and inconsistent product descriptions. Some descriptions include partial parameter information, such as " 2 ", black." Others focus on how the product might be used, and still others are highly terse and omit most details.

A second challenge is the difficulty of matching the buyer's search terms to the wording in the descriptive fields. When exact term matches don't exist, the search must consider issues such as word stemming, spelling errors, abbreviations, and synonyms.

A third challenge is that although product descriptions represent numeric attributes poorly and incompletely, some users will nonetheless want to search by size, weight, height, and so on. Every catalog offers many products, the product lines are continually changing, and each of the thousands of product categories has a different set of attributes. As a result, 
catalogs do not store attributes as fields of a table. Rather, they store attribute names and their value settings as data items; this makes searches more difficult.

Shopping catalogs often include many dozens, even thousands, of tables to describe the products being offered and to support services and user needs. To improve runtime performance, these sites often use a denormalized field called a munge as the search engine target. This munge places into its subfields copies of each of the catalog's searchable fields, such as item name, item ID, category name and ID, model or part number, description, price, maker, condition, and all attributevalue pairs. In effect, the munge is like a document on each product in the catalog.

Because most search algorithms can't infer the subfields to search, they search the entire munge. For example, a keyword search searches across all subfields of the full munge for a strict match on terms. CQ traverses the same ground but can also look for synonyms, alphabetically similar terms, and related conceptualizations. NLQ is the only search strategy that infers the labels or field names of each token in the query string. Hence, it can then send the (conceptual) search to the precise subfields of the catalog in which the query's tokens should exist, provided they are in the database.

The reason that CQ does worse than NLQ is that it must deal with term semantics and ambiguities without a parser. NLQ, on the other hand, can parse the entire query string, label its tokens, and hold an interactive conversation about the query to confirm its interpretation. NLQ researchers argue that CQ languages would benefit from the addition of a parser with a grammar restricted to the database's domain. Although such parsers have less-than-general interpretative power, they can still improve trouble management and help the interface conform to the user's language. Several systems in the lab purport to provide these extensions, ${ }^{3-6}$ and some offer formal slotted grammars (with semantically typed slots) for merging the natural language approach with CQ.

Proponents of pure CQ, in turn, argue that natural language extensions tend to be impractical: indeed, none of the NLQ systems just cited has been evaluated on a large scale. The CQ people argue that the "natural language problem" is too difficult and remains unsolved on any reasonable scale. As a result, most large-scale relational database management products (for example, Oracle, Sybase,

All query languages attempt to match a query string to database entries. Readers interested in a survey of the many approaches should consult the references; ${ }^{1-5}$ here, we compare two of them.

\section{Conceptual query}

Literally, this refers to an expansion of the search terms with the help of a concept tree of closely related terms. However, in practice, this also includes any other expansion, such as using a thesaurus to generate synonyms. Generally, prior to the expansion, the conceptual query search tokenizes the search string and removes stop words. Then, it passes the tokens to traditional Structured Query Language for matching against the database entries.

\section{Natural language query}

In addition to all the steps of conceptual query, NLQ includes a parser that attempts to identify and label the part of speech of each token in the search string. Such labels can reduce the expansion set to entries using words in the same sense as the original and help the traditional SQL narrow its search.

\section{References}

1. C.J. VanRijsbergen, Information Retrieval, Butterworth, London, 1979.

2. S.M. Dekleva, "Is Natural Language Querying Practical?" Data Base, vol. 25, no. 2, 1994, pp. 24-36.

3. M.J. Bates, "Indexing and Access for Digital Libraries and the Internet: Human, Database, and Domain Factors," J. Am. Soc. Information Science, vol. 49, no. 13, 1998, pp. 1185-1205.

4. K. Sparck-Jones and P. Willett, Readings in Information Retrieval, Morgan Kaufmann, San Francisco, 1997.

5. H. Cunningham, Information Extraction, a User Guide, tech. report CS-99-07, Dept. Computer Science, Univ. of Sheffield, Sheffield, UK, 1999; www.dcs.shef.ac.uk/ hamish/IE/userguide/main.html (current 26 July 2001).

DB II, or AltaVista) that are widely used by market exchanges and other large-scale ecommerce sites include CQ features for those who choose to deploy them, but exclude NLQ. Some smaller, less formally defined NLQ systems appear to work atop specific databases or environments. ${ }^{7,8}$

Certain e-commerce Web sites have deployed natural language self-help or chatterbots, but these bots handle site navigation and document retrieval issues and cannot process catalog or database search requests. This inability adds fuel to the arguments that only CQ can scale up to the e-catalog task. ${ }^{4}$

In sum, no examples exist of NLQ working in large-scale e-commerce catalog shopping sites, and it is tempting to believe the proponents of CQ rather than NLQ. The research results we report here are the first large-scale test offering evidence to counter pure-CQ proponents. Our results show that NLQ is an effective complement to CQ in shopping Web sites.

\section{System architecture and algorithm}

Now let's take a look at how our agent, called EQUIsearch, performs NLQ. We assume that a Markov decision process is suitable to analyze the semantics and morphosyntactics of the user's query. Specifically, at any moment in time, the agent is in one of a finite number of states $(\mathrm{s}=1, \mathrm{~S})$ and must choose one of a finite set of actions (a $=1, \mathrm{~A})$ to transition to the next state. More specifically, optimizing a Markov decision process is a dynamic programming problem that maximizes $E(U)$, the expected discounted rewards across future periods, as

$$
\operatorname{Max} \mathrm{V}^{*}=\mathrm{E}\left[\sum_{\mathrm{t}=1}^{\mathrm{T}} \mathrm{U}\left(\mathrm{s}_{\mathrm{t}}, \mathrm{a}_{\mathrm{t}}\right)\right] \text {, }
$$

where $\mathrm{V}^{*}$ is the optimum value point (in terms of precision and recall) and $U($ ) is the reward function or utility from selecting action $\mathrm{a}_{\mathrm{t}}$ at state $\mathrm{s}_{\mathrm{t}}$.

We can solve Equation 1 if we loop across iterations $(\mathrm{t}=1, \mathrm{~T})$ and for each iteration loop across all states $(\mathrm{s}=1, \mathrm{~S})$ to find the action or set of actions that maximizes both current and future rewards to avoid local optima. We capture this expansion by finding the maximal value of the following function after testing all possible actions, $\mathrm{a}=1, \mathrm{~A}$ : 


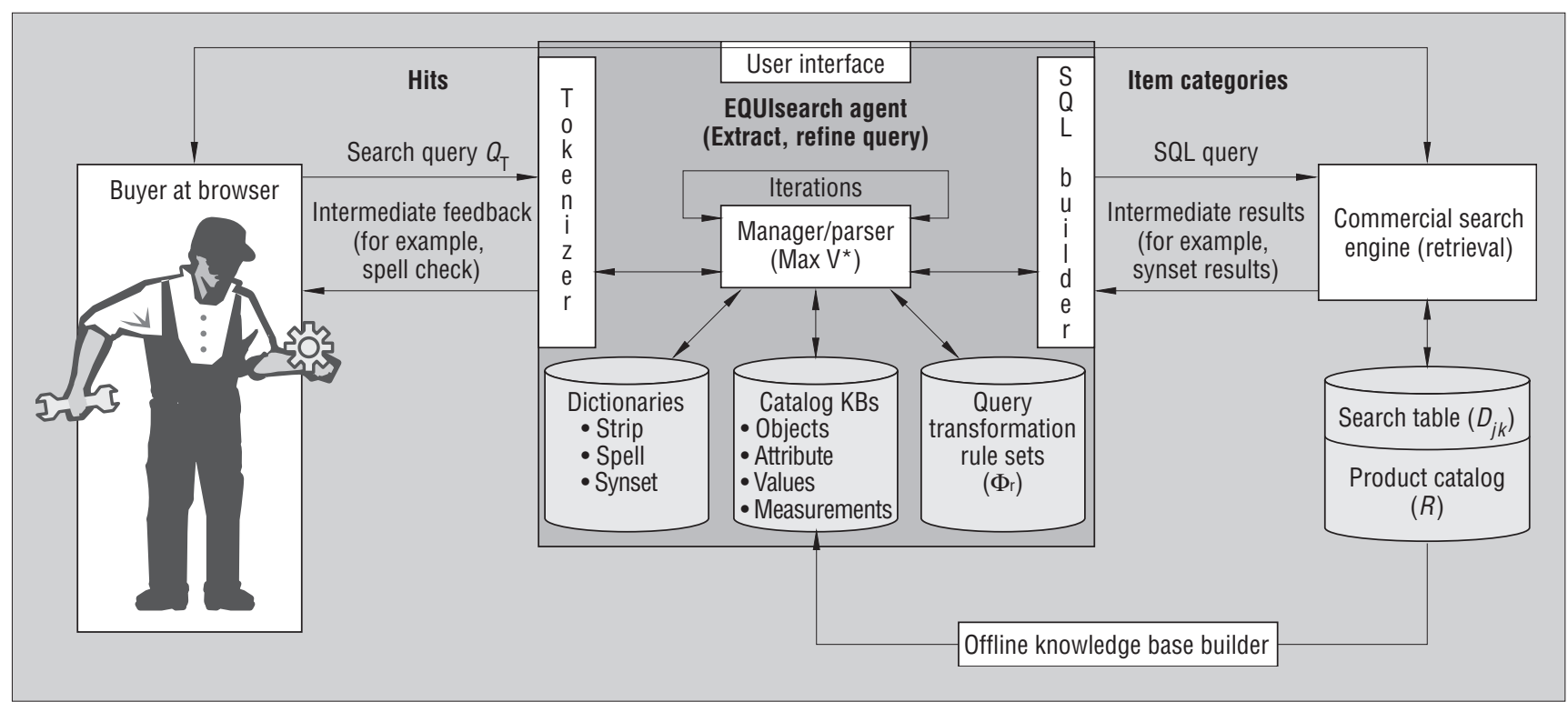

Figure 1. Overview of the EQUlsearch agent as a meaning translator in the interface between users and traditional search engines.

$$
\begin{aligned}
& \mathrm{Z}_{\mathrm{t}}(\mathrm{s}, \mathrm{a})=\mathrm{U}\left(\mathrm{s}_{\mathrm{t}}, \mathrm{a}_{\mathrm{t}}\right)+ \\
& \delta \sum_{\mathrm{S}_{\mathrm{t}+1}=1}^{\mathrm{S}} \pi\left(\mathrm{s}_{\mathrm{t}}, \mathrm{a}_{\mathrm{t}}, \mathrm{s}_{\mathrm{t}+1}\right) \mathrm{V}_{\mathrm{t}-1} *\left(\mathrm{~s}_{\mathrm{t}} .\right.
\end{aligned}
$$

Here, $\pi\left(\mathrm{s}_{\mathrm{t}}, \mathrm{a}_{\mathrm{t}}, \mathrm{s}_{\mathrm{t}+1}\right)$ is the transition probability of being in state $s_{t}+1$ immediately after taking action $\mathrm{a}_{\mathrm{t}}$ from state $\mathrm{s}_{\mathrm{t}} \cdot \mathrm{V}_{\mathrm{t}-1} *\left(\mathrm{~s}_{\mathrm{x}}\right)$ is $\mathrm{Z}_{\mathrm{t}+1}$ $\left(\mathrm{s}_{\mathrm{t}+1}, \operatorname{argmax} \mathrm{a}_{\mathrm{a}}\left(\mathrm{Z}_{\mathrm{t}-1}(\mathrm{~s}, \mathrm{a})\right)\right.$, where $\operatorname{argmax}_{\mathrm{a}}$ finds the maximal action.

Thus, recursive Equation 2 summarizes the standard computable value iteration formulation. To use this, we must define the permissible states and actions, the reward function, the transition probabilities, and other terms of the equation for catalog search problems. We provide an overview of these items in what follows and in Figure 1; readers can find the details elsewhere. ${ }^{9}$

As a language parser, the agent iteratively labels each stripped and stemmed token of the query. Then, it modifies these labels by applying a sequence of transformation rules to reduce the remaining ambiguities and residual errors left by the previous rules. In doing so, it labels the state of each term in the query, where state is defined within a subgrammar. The subgrammar we created here is called OAV Triplet Grammar. OAV triplets, or object-attribute-value triplets, are metadata that describe what catalogs contain in much the same way as resource description formats (RDFs) describe the Web's content. ${ }^{10,11}$

Specifically, in product catalog domains, users usually search for objects with attrib- utes of a certain value. This corresponds to searching for a noun phrase that includes adjectives. For example, some triplets in various orderings might be "mini sized amps" or "hammer colored red." The agent must discover the order of the triplets. Thus, a common order variant occurs where the agent initially seeks the object (the $\mathrm{O}$ in $\mathrm{OAV}$ ) and then uses the AVs for comparison and search refinement-for example, bolt cutter followed by size, price, and availability. Even more common are searches for one or more $\mathrm{V}$ of a given type of $\mathrm{O}$ where the $\mathrm{A}$ is suppressed-for example, AA Eveready batteries, 1/2" no. 8 slotted screws, or desk chair. (In the last example, the first of the two consecutive nouns functions as an adjective.)

In addition to the subgrammar's rules, we obviously must also derive a vocabulary for any given instantiation or catalog. In shopping domains, we can derive the vocabulary for objects and another for attribute-value pairs by crawling the relational catalog database's fields and extracting all unique terms: item names are object name, attributes are parameter names, and parameter settings are attribute values. We show this tool for crawling the catalog and extracting the knowledgebase values at the bottom of Figure 1. Using such a tool, we can readily construct the subgrammar and its vocabulary for any given electronic catalog. The KB lookup tables (at the base of the agent in Figure 1) store the result so the phrase parser's transformation rules can use it to infer and insert $\mathrm{O}, \mathrm{A}$, and
$\mathrm{V}$ labels onto the various terms of any given search phrase. At the bottom right of the agent box, Figure 1 shows other rules $\left(\Phi_{\mathrm{r}}\right)$ that the agent uses to infer OAV meanings from queries (for example, the rule about consecutive nouns).

It is not sufficient that the catalog's extracted vocabulary covers 100 percent of a given shopping site's lexicon if the site's users are unaware of that lexicon or prone to misusing it. To help overcome such semantic impasses, the agent first stems the tokens in the query string, expands them for synonyms, and ultimately checks them for spelling (see bottom left of the agent in Figure 1). The dictionaries and KBs across the base of Figure 1 support these tasks.

\section{Comparison testing EQUlsearch}

In Figure 1, we showed EQUIsearch as complementing commercial search engines for online catalogs. To illustrate that point, we empirically evaluated query performance both with and without the EQUIsearch agent for a major commercial search engine relevant to e-commerce catalogs.

In general, there are four ways to measure search engine performance: retrieval effectiveness metrics (such as recall and precision), user satisfaction measures, transaction $\log$ analysis, and the critical incident technique. Another article examines the last three of these. ${ }^{12}$ In this study, we used effectiveness metrics for our comparison. 
Table 1. Contingency table for deriving effectiveness metrics.

\begin{tabular}{lll} 
& $\begin{array}{l}\text { Pertinent } \\
\mathbf{P}=\mathrm{RP}+\mathrm{NP}\end{array}$ & $\begin{array}{l}\text { Irrelevant } \\
\mathbf{I}=\mathrm{RI}+\mathrm{NI}\end{array}$ \\
$\begin{array}{l}\text { Retrieved } \\
\mathbf{R}=\mathrm{RP}+\mathrm{RI}\end{array}$ & $\mathbf{R P}$ & $\mathbf{R I}$ \\
\hline & Hits & Type I errors \\
\hline $\begin{array}{l}\text { Not retrieved } \\
\mathbf{N}=\mathrm{NP}+\mathrm{NI}\end{array}$ & $\mathbf{N P}$ & $\mathbf{N I}$ \\
\hline
\end{tabular}

Total catalog $=\mathrm{RP}+\mathrm{RI}+\mathrm{NP}+\mathrm{NI}$ appropriateness. Ultimately, the user determines whether a document is pertinent; in this case, we do. The ubiquitous contingency table, Table 1 in this article, delineates the categories for retrieved and unretrieved items in a catalog.

For our purposes, we will use ratios of hits/retrieved and hits/pertinent. We define the ratio of hits to items retrieved as precision. We term the ratio of hits to pertinent items the recall. From Table 1, we see that

$$
\text { Precision }=\mathrm{RP} /(\mathrm{RP}+\mathrm{RI})
$$

and

$$
\text { Recall }=\mathrm{RP} /(\mathrm{RP}+\mathrm{NP}) .
$$

\section{Scale-up test results}

To test whether NLQ can scale up, we deployed it at a market exchange. EqualFooting (www.equalfooting.com) is a B2B online marketplace for the maintenance, repair, and operations (MRO) sector. ${ }^{12}$ Basically, this means that EqualFooting sells industrial and construction supplies-something like a Home Depot for small contractors, but with an order of magnitude more products. The company's official launch date was February 2000 , and by June 2000 it was handling one million hits per day (by about 23,000 separate users daily). At this writing, EqualFooting's catalog integrates almost 450,000 products from more than 2,000 sellers.

The company stores its catalog internally in an Oracle database. Users at this Web site now use the EQUIsearch NLQ agent, which in turn interfaces with Oracle and its CQ search technology, Oracle interMedia.

The conceptual search features of products such as interMedia are not immediately usable at a given shopping site. For example, making the production version of the EqualFooting database CQ-capable required creating the three dictionaries always necessary for conceptual search — spelling, stripping, and synonyms - because these are domainspecific items.

Growing the thesaurus involved many false starts and deadends. For example, we were tempted to use an existing general-purpose thesaurus such as WordNet from Princeton, which includes 95,000 words and all their synonyms. However, this thesaurus brings

back too many synonyms, many of which are inappropriate (racial slurs, curses, body parts, religious terms, and so on). In addition, it omits most of a given domain's specialty terms. For example, chain saw, Phillips head, and safety gloves are among the thousands of items found in a hardware catalog that have no entries in WordNet. Instead, based on search log analyses, EqualFooting assembled its own thesaurus for almost 8,000 synonyms critical to its domain, although still more synonyms are needed.

The second dictionary task was to give the database a spell checker. Oracle doesn't ship interMedia with this function, so the company purchased one and installed it separately. Although it came with 100,000 words, EqualFooting embellished the spell checker's dictionary by adding

- the top 1,000 misspelled words from the user search logs and their corrections,

- proper names of all manufacturers and suppliers (the spell checker initially assumed that all proper names were errors) and possible misspellings, and

- many hundreds of acronyms with proper spellings (such as CD, DVD, HVAC, and so on).

The third and final dictionary task was to massage the stop word list for the MRO domain. Some generic stop words were relevant here (for example, AND, THE, ALL), but others are unique (such as $\mathrm{X},-$, , and /).

For a site that has already prepared the three dictionaries CQ requires (spelling, stripping, and synonyms), the extra effort to add EQUIsearch is rather straightforward:

1. Run a catalog crawler that extracts all the lexicon.

2. Construct the lexical knowledge bases (objects and attribute-value pairs).

3. Author the relevant rule sets and encode them in the $\Phi_{\mathrm{r}}$.

4. Complete any interface code needed in the agent's SQL builder to adapt it to the requirements of the CQ technology (interMedia in this case).
We performed these steps manually for the test site during the fall of 2000 and deployed the agent on 17 November 2000. Since then, it has been in continuous operation-24 hours, 7 days a week.

\section{Comparison test results}

For comparison testing, we ran three types of query strings as listed in Table 2. Nouns or objects are the item names or synonyms of those names. Noun-adjective pairs (objectattribute pairs) cover cases in which the user seeks to narrow the choices returned. Lastly, although most users are unaccustomed to using sentence format, we thought it was an important search category because we hope future users will be unaware of search engines' previous constraints.

Statistics on current search habits indicate that users type about 2.3 words per query, with nouns being the most common search, noun-adjective pairs next most common, and multiword phrases being least common. ${ }^{1,2}$ We designed our test bank, shown in Table 2 , to approximate this distribution of 2.3 words per query on average: 146 words $\div 63$ queries $=2.3$ words/query.

Table 3 compares effectiveness and timing statistics for the CQ search (via Oracle interMedia) with those for the NLQ agent. We collected these results over EqualFooting's intranet, so they don't include Internet latency, but that would be identical for both methods. Inspecting the last row of Table 3 shows that, on average, the CQ search was nearly twice as fast as NLQ ( 0.5 versus 0.9 seconds per query). However, CQ tended to retrieve nearly three times as many hits, although its recall was not as good as NLQ's $(0.8$ versus 1.0$)$ and its precision was only half as good (0.5 versus 1.0). Furthermore, the time advantage of CQ search is relatively inconsequential, because most users can't detect a half second of difference.

Consider also the first row of the Table 3 , noun search, where we see NLQ holding a clear advantage across the board. Not only is NLQ more precise, it's also faster. The only place CQ holds its own is in recall, where both methods are perfect in bringing back all 
Table 2. Query strings used to comparison test CQ and NLQ.

\begin{tabular}{|c|c|c|}
\hline Nouns (31) & Noun-adjective pairs (16) & Sentences (14) \\
\hline Aircompressor & 16 oz hammer & Show me all power cords. \\
\hline Ballast & 10 inch nail & List me cotton gloves. \\
\hline Blower & Bolt cutter & Show me gloves made of leather. \\
\hline Brad & Copier paper & I want to buy leather gloves. \\
\hline Brush & Cotton glove & List me nails for roofing. \\
\hline Cabinet & Crimped brush & List me all wheel cutters. \\
\hline Calculator & Cutter wheel & Show me bolt cutter. \\
\hline Chipper & Leather glove & List wire connectors. \\
\hline Chisel & Nail hammer & List brush made of steel. \\
\hline Cleaner & Pipe clamp & I want to buy a crimped brush. \\
\hline Compressor & Power cord & Let me see leather gloves if you have any. \\
\hline Connector & Protective apron & \\
\hline Cupboard & Roofing nail & \\
\hline Cutter & Safety tape & \\
\hline Drill & Steel brush & \\
\hline Fans & Tape measure & \\
\hline Gloves & White paper & \\
\hline Grinder & Wire connector & \\
\hline \multicolumn{3}{|l|}{ Hammer } \\
\hline \multicolumn{3}{|l|}{ Ladder } \\
\hline \multicolumn{3}{|l|}{ Mallet } \\
\hline \multicolumn{3}{|l|}{ Mitten } \\
\hline \multicolumn{3}{|l|}{ Nail } \\
\hline \multicolumn{3}{|l|}{ Paper } \\
\hline \multicolumn{3}{|l|}{ Pipe } \\
\hline \multicolumn{3}{|l|}{ Processor } \\
\hline \multicolumn{3}{|l|}{ Saw } \\
\hline \multicolumn{3}{|l|}{ Screw } \\
\hline \multicolumn{3}{|l|}{ Snapper } \\
\hline \multicolumn{3}{|l|}{ Soap } \\
\hline Tube & & \\
\hline
\end{tabular}

the pertinent items.

This story changes for noun-adjective pair search (row 2), at least in terms of speed. Here, NLQ takes almost four times as long as CQ, although it still wins on precision. It should surprise no one that the NLQ is faster than CQ in noun search and slower in noun- adjective search. In noun or object search, CQ must dynamically sort and index the entire munge (many subfields denormalized), whereas NLQ need only sort through the item-name field, because it narrows the search by labeling the token. Likewise, in object-attribute search, CQ behaves the same as before, but NLQ causes Oracle interMedia to sort an additional set of fields (all those with attributes) and then do a soft join and eliminate items not in all parts of the join.

NLQ handles sentences (row 3) more quickly than word pairs (row 2), further confirming that the parsing processes are not very significant determinants of delay. A query's specific terms drive the process. In this case, the noun-adjective pairs that the parser extracted from the sentences proved to be less common items in the database; hence the Oracle $\mathrm{CQ}$ engine could process them faster.

\section{Results analysis}

We intend the NLQ agent presented in this article to complement and improve search engines for online e-commerce shopping catalogs. Our results show that the agent improves the precision and recall of the search with no significant overall impact on response time. Several of the lessons we learned in deploying and testing EQUIsearch bear further discussion:

- Scaling up NLQ. To scale up NLQ, we did away with some of the less practical NLQ proposals in the literature, such as conversational feedback and explaining the query translation to be used prior to executing the query. Instead, EQUIsearch converses with the user in the fashion that CQ systems use: after the query, by displaying either hits or canned, limited explanations of query failures. Also, we implemented our agent as a Markovian decision process. The agent continues to work at a relatively large-scale market exchange, which previously operated with CQ search alone.

- CQ paves the way for domain-specific $N L Q$. Relational DBMSs ship with CQ features, but each enterprise must enable and fill in the stripping, synonym, and spelling dictionaries before CQ can work for its shopping catalog. The good news is that these are the same three dictionaries

Table 3. Timing and effectiveness results for CQ search versus the NLQ agent.

\begin{tabular}{|c|c|c|c|c|c|c|c|c|}
\hline \multirow[t]{2}{*}{ Search } & \multicolumn{2}{|c|}{ Total retrieved } & \multicolumn{2}{|c|}{ Precision } & \multicolumn{2}{|c|}{ Recall } & \multicolumn{2}{|c|}{$\begin{array}{l}\text { Two-run average time } \\
\text { (seconds) }\end{array}$} \\
\hline & $C Q$ & $N L Q$ & $\mathrm{CQ}$ & $N L Q$ & $C Q$ & $N L Q$ & $C Q$ & $N L Q$ \\
\hline $\begin{array}{l}31 \text { nouns } \\
18 \text { noun-adjective pairs } \\
14 \text { sentences } \\
\text { Average (for } 63 \text { queries) }\end{array}$ & $\begin{array}{r}2,965 \\
160 \\
1 \\
1,580\end{array}$ & $\begin{array}{r}1,091 \\
66 \\
71 \\
571\end{array}$ & $\begin{array}{l}0.6 \\
0.6 \\
0.0 \\
0.5\end{array}$ & $\begin{array}{l}1.0 \\
1.0 \\
1.0 \\
1.0\end{array}$ & $\begin{array}{l}1.0 \\
1.0 \\
0.0 \\
0.8\end{array}$ & $\begin{array}{l}1.0 \\
1.0 \\
1.0 \\
1.0\end{array}$ & $\begin{array}{l}0.6 \\
0.5 \\
0.4 \\
0.5\end{array}$ & $\begin{array}{l}0.4 \\
1.9 \\
0.8 \\
0.9\end{array}$ \\
\hline
\end{tabular}


that NLQ needs. If a site has already developed them and deployed CQ, it takes only minimal extra effort to deploy NLQ.

- Data-cleansing obstacles remain for any search method. Numerous typos, missing item names and other data, and poorquality attribute information constitute possibly the most serious obstacle to scale up a search in any unified shopping cata$\log$. This obstacle is not unique to NLQ, but equally plagues CQ and keyword search methods.

- Speed differences are irrelevant in most cases. It seems that NLQ is faster for noun search, but slower than CQ for noun-attribute pair searching. But in either case, the time differences are not statistically significant.

- NLQ agent provides precision and recall improvement. Our results to date reflect about a 50 percent improvement in precision when NLQ is added to CQ. This means that users experience shortened retrieval sets and that the items retrieved include far fewer false positives. Additionally, there are fewer false negatives or relevant items omitted.

- NLQ agents offer parsing services $C Q$ can't provide. Many shopping sites have begun to add chatterbots, such as Dell's AskDudley (http://support.dell.com/us/ en/askdudley), that provide navigation help and answer site or content questions in natural-like language. The results to date indicate that users like this type of self-service help. When it is present, they build up a higher expectation that the catalog search will behave in a similarly naturalistic way, and they no longer limit their queries to the short keyword format (the 2.3-word average mentioned earlier). ${ }^{2}$ They pose English-like sentences and questions to the catalog search engine, and it seems they are frustrated by the CQ search engines' inability to parse their questions. NLQ agents such as EQUIsearch appear to be the answer for shopping sites facing this dilemma.

$\mathbf{T}$ he difficulty of searching online shopping catalogs is the difficulty of the Web in general: content created for human consumption poses a challenge for machine interpretation and for use by other agents. To address this larger challenge, DARPA and the $\mathrm{W} 3 \mathrm{C}$ have significant efforts under way to create the Semantic Web-tagging the semantics of the Web so that content created by laypersons for use by other people is auto- matically and transparently marked up for machine and agent interpretation and use. The process draws on tools such as the W3C's RDF, the DARPA agent markup language (DAML), and the ontology interface language (OIL) ${ }^{10,11}$ Although we did not use this tool set in the work we report here, having it available and scaled up for industrial use could integrate catalogs into the Semantic Web. Potentially, this could ease the content interpretation of market catalogs.

The Semantic Web efforts do not include research on NLQ, so it would also be interesting and challenging to explore how to extend natural language agents such as those we describe in this article to work in the broader domain of the Semantic Web. In general, such agents operate in narrow domains only, so the challenge would be to populate the Web with many such special-purpose agents.

\section{References}

1. "Winning the Online Consumer: Insights Into Consumer Behavior," Boston Consulting Group, Cambridge, Mass., 2000, www.bcg. com (current 3 July 2001).

2. P.R. Hagen, H. Manning, and Y. Paul, "Must Search Stink?" Forrester Research, Cambridge, Mass., 2000, www.forrester.com (current 3 July 2001).

3. V. Owei, "Natural Language Querying of Databases: An Information Extraction Approach in the Conceptual Query Language," Int'l J. Human-Computer Studies, vol. 53, 2000, pp. 439-492.

4. S.M. Dekleva, "Is Natural Language Querying Practical?" Data Base, vol. 25, no. 2, 1994, pp. 24-36.

5. M.T. Pazienza, ed., Information Extraction: Toward Scalable, Adaptable Systems, Springer-Verlag, Berlin, 1998, pp. 95-119.

6. T. Strzalkowski, Natural Language Information Retrieval, Kluwer Publishing, Dordrecht, Netherlands, 1999.

7. A. Blum, "Add Natural Language Search Capabilities to Your Site with English Query," Microsoft Interactive Developer, Apr. 1998, www.microsoft.com/Mind/0498/equery.htm (current 28 June 2001).

8. "Revolutionizing the Search for Products at E-Commerce Sites," Easy Ask Inc., Littleton, Mass., Mar. 2000, www.easyask.com/ technology/pdf/revolutionizing_search.pdf (current 3 July 2001).

9. B.G. Silverman et al., "A Markov Decision Processing Solution to Natural Language Querying of Online e-Commerce Catalogs," submitted to J. OR in Computing, available from http://www.seas.upenn.edu/ barryg/ mdp.pdf.

10. J. Hendler, "Agents and the Semantic Web," IEEE Intelligent Systems, vol. 16, no. 2, Mar./Apr. 2001, pp. 30-37.

11. M. Klein, "XML, RDF, and Relatives," IEEE Intelligent Systems, vol. 16, no. 2, Mar./Apr. 2001, pp. 26-28.

12. B.G. Silverman et al., "Buyer Decision Support Systems and Search Agents for eCommerce Websites," to be published in Int'l J. Human-Computer Studies, vol. 54.

13. C.J. VanRijsbergen, Information Retriveral, Butterworth, London, 1979.

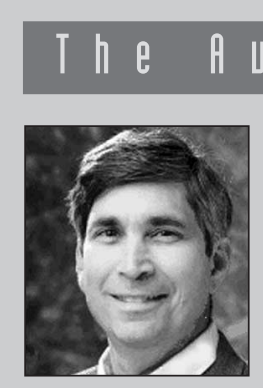
thors Advancement of Systems Approaches. He is a fellow of the IEEE and AAAS. Contact him at Towne Bldg, Rm 229c, University of Pennsylvania, Philadelphia, PA 19104-6315; barryg@ @eas.upenn.edu, http://www.seas.upenn. edu/ barryg.

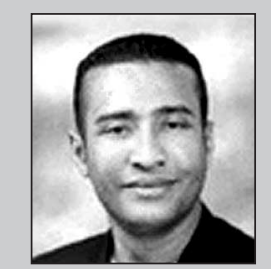

Mintu Bachann is both $\mathrm{CIO}$ and $\mathrm{COO}$ of Equidity, which offers full-service business financing technology, from loan origination to fulfillment. Prior to Equidity, Bachann worked on technology infrastructure and architecture for organizations such as NationsBank, Oracle, Bank of America, Barnett Bank, and Sun Microsystems. Bachann holds a doctorate of science in artificial intelligence and computer science from George Washington University.

Khaled Al-Akhras is Vice President of Engineering for Equidity. Previously, he worked in the software design and development industry, where he designed real-time decision support systems and simulators for nuclear power utilities all over the world. He holds a master's degree in computer science from George Washington University. 